\title{
NON-SCANNING MAGNETIC FIELD IMAGING WITH LASER-PUMPED ATOMIC MAGNETOMETER
}

\author{
Lebedev $\mathrm{V}^{1}$, Dolgovskiy $\mathrm{V}^{1}$, Michen $\mathrm{B}^{2}$, Fink $\mathrm{A}^{2}$, Bison $\mathrm{G}^{1}$, Weis $\mathrm{A}^{1}$ \\ ${ }^{1}$ Department of Physics, University of Fribourg, Switzerland \\ ${ }^{2}$ Adolphe Merkle Institute and Department of Chemistry, University of Fribourg, Switzerland \\ ${ }^{3}$ Paul Scherrer Institute, Villigen, Switzerland
}

victor.lebedev@unifr.ch

\begin{abstract}
We present first results on the imaging of the twodimensional magnetic field distributions using a recently developed magnetic field imaging camera (MFIC). The instrument is based on laser-pumped atomic magnetometry with an alkali vapour in a buffer gas. The device provides millimetre spatial and sub-second time resolution, and allows mapping the magnetic field from $\approx 2 \mathrm{~cm}^{2}$ surfaces. We apply the MFIC to image magnetic field patterns of deposited superparamagnetic nanoparticles (SPIONs) and quantify the achieved sensitivity. We address the applicability of the instrument to in-vitro and in-vivo imaging of SPIONs distributions in biological tissue and small animals.
\end{abstract}

Keywords: magnetic imaging, atomic magnetometer, SPIONS

\section{Introduction}

During the past two decades, magnetic nanoparticles have found numerous biomedical applications for diagnostics and therapy. Superparamagnetic nanoparticles (SPIONs) are used as contrast agents in MRI, and are studied in view of applications to hyperthermia, drug delivery and cell targeting [1]. Many of those applications demand for a non-invasive technique that allows the high spatial resolution detection of SPION distributions in the tissue or organism in order to improve the treatment efficiency and to avoid negative side effects.

Laser-pumped atomic magnetometers (LM) are known as compact, high sensitivity, and practically service-free magnetic field sensors that operate near room temperature. They are currently considered as alternatives for SQUIDbased magnetometers in many applications. In an LM, the magnetic field information is retrieved by a weak laser beam traversing the sensing element, an atomic vapour cell. Buffer gas filled cells with immobilized atoms combined with video-imaging of laser light offer the possibility to realize a simple sensor module capable to directly detect magnetic field distributions at the centimetre scale. The sensitivity of such a detector is inversely related to its spatial resolution and image recording time. We believe, however, that optimised LMs will have the required sensitivity for detecting SPIONs with bio-compatible densities and millimetre space resolution.

\section{Methods}

The operating principle of an atomic magnetometer is based on the high sensitivity of the atomic spin polariza- tion in an ensemble of alkali metal atoms to the ambient magnetic field [2]. Spin polarization, and hence a macroscopic magnetization is achieved by the technique of optical pumping, whereby the irradiation of the sample by laser light of specifically chosen wavelength, direction and polarization transfers angular momentum to the atomic ensemble. The same (or a second suitable) laser beam transmitted through the sensor cell reads out the sample's magnetization that is affected by the external magnetic field at the sensor position. Information on the local field can be inferred from the intensity and/or polarization of the transmitted laser beam.

In our MFIC design, the sensing caesium atoms are slowly diffusing in a sufficiently dense inert buffer gas, with positions defined at the level of several ten $\mu \mathrm{m}$ on the ms time scale that is sufficient to polarize and read out the atomic spins. By using expanded laser beams and a sensitive video camera as light detector (Fig. 1) we achieve in this way a mapping of the spin polarization and hence of the magnetic field distribution over the whole illuminated volume.

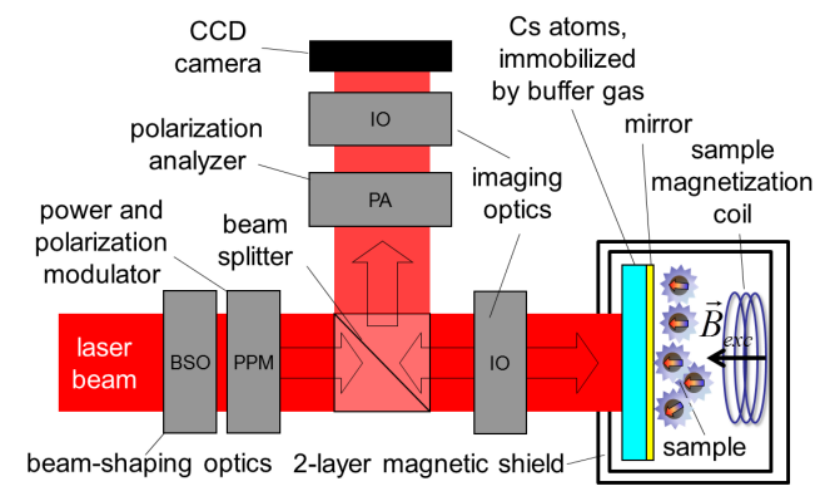

Figure 1: Design of the magnetic field imaging camera.

In the presented experiments we studied the magnetometric sensitivity and spatial resolution in images of finestructured SPION patterns deposited on a glass substrate with an average density of $20 \mathrm{mg} / \mathrm{cm}^{2}$. The sample is magnetized by a mT-strong magnetic field pulse and the field pattern is recorded during the magnetization decay.

\section{Results}

Figures of merit for this study are the integral magnetic sensitivity of the sensor (IS), closely related to the quanti- 
ty of the SPIONs detectable in a single measurement, as well as the effective spatial resolution of the camera (ESR).

At BMT 2012, we have shown by a time-resolved atomic diffusion study in a cubic sensor cell [3] that we can reach an ESR of $1.5 \mathrm{~mm}$. This value defines a reasonable binning for the video camera to produce a $10 \times 10$ element magnetic image resolution for our $15 \times 15 \mathrm{~mm}^{2}$ sensor cell. The IS can be understood as the magnetic field detection limit when the camera image is used as a single pixel detector. In a moderately magnetically shielded environment that cancels the detrimental effects of ambient ac and dc magnetic fields, we reach an IS that is equivalent to 0.7 nT with a one second averaging time. This value translates into a $200 \mu \mathrm{g}$ SPION detection limit with single frame recording and $25 \mu \mathrm{g}$ with one second averaging. For an image of $10 \times 10$ bins, the latter value corresponds to $\approx 2.5 \mathrm{mg}$ of SPIONs per bin, equivalent to $5 \mathrm{mg} / \mathrm{mm}^{2}$. These numbers are supported by experiments on SPIONs patterns.

\section{Discussion}

The demonstrated sensitivity is obviously far from the needs of medical applications that demand for a few $\mu \mathrm{g}$ of nanoparticles in the whole sample. However, our current experiments are strongly limited by the performance of the video camera, the fast decay of the sample magnetization during the measurement time, and ambient magnetic field noise.

In fact, our current useful signal range is limited to approximately two percents (4 bit) of the camera's dynamic range. Tests with a discrete photodiode replacing the camera have yielded a sensitivity that surpasses the above values by more than two orders of magnitude (at the same exposure time). Furthermore, the slow data rate of the camera leads to a poor usage of the measurement time, in which a ms-long signal exposure (needed to maximize spatial resolution) is followed by $8 \mathrm{~ms}$ image data transfer time. Replacing the imaging sensor with a high speed InGaAs camera or an array of discrete photodiodes offers a straightforward solution to those issues.

Another drawback of the studies carried out so far is the use of a commercial large volume $\left(15^{3} \mathrm{~mm}^{3}\right)$ cubic quartz glass sensor cell that imposes geometrical limits on the image resolution. We are working on overcoming this issue by the implementation of a (quasi two-dimensional) MEMS cell that is expected to bring the ultimate resolution to a diffusion-limited level below $100 \mu \mathrm{m}$.

The mentioned, but technically demanding modifications of the MFIC are under current development and we will present a status update at the conference.

\section{Acknowledgement}

Work supported by Sinergia grant No. CRSII2_144257 of the Swiss National Science Foundation.

\section{Bibliography}

[1] Pankhurst Q.A., Conolly J. et al.: Applications of magnetic nanoparticles in biomedicine. J. Phys. D: Appl. Phys., Vol. 36, No. 13, pp. 167-181, 2013.

[2] Budker D. and Romalis M.: Optical magnetometry, Nature Physics, Vol. 3, pp. 227-234, 2007.

[3] Lebedev V., Castagna N. et al.: Imaging of magnetic nanoparticles by atomic magnetometers, BMT 2012 $46^{\text {th }}$ annual conference of the German Society for Biomedical Engineering, Jena, September 16-19, 2012. 